\title{
Attempt to accelerate asymmetric species with unequal frequencies in RHIC
}

\author{
C. Liu, Y. Luo, A. Marusic, M. Minty, \\ G. Robert-Demolaize, K. Smith, \\ K. Mernick, T. Hayes, F. Severino
}

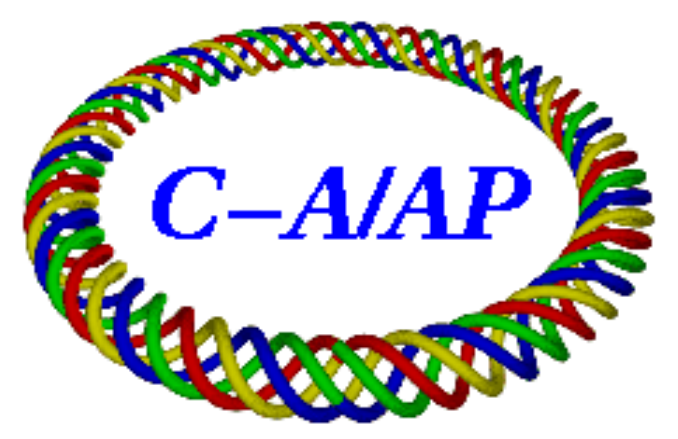

\section{Collider-Accelerator Department Brookhaven National Laboratory Upton, NY 11973}

\section{U.S. Department of Energy}

Office of Science, Office of Nuclear Physics 


\title{
Attempt to accelerate asymmetric species with unequal frequencies in $\mathrm{RHIC}^{*}$
}

\author{
C. Liu, Y. Luo, A. Marusic, M. Minty, G. Robert-Demolaize, \\ K. Smith, K. Mernick, T. Hayes, F. Severino, \\ Brookhaven National Lab, Upton, NY, U.S.A.
}

July 9,2015

\begin{abstract}
This report summarizes the beam studies on accelerating asymmetric beams with unequal frequencies, during the proton-Gold/Aluminum run in 2015. The experiment failed due to modulated beam-beam effects even though the beams were separated by at least $15 \mathrm{~mm}$.
\end{abstract}

\section{Introduction}

There have been a couple of asymmetric runs in RHIC [1, 2, 3]. The first one is the 10 weeks deuteron-gold collision in 2003. During the 2003 operation beams were initially injected with the same rigidity and therefore different revolution frequencies at injection and the majority part of the ramp. This led to unacceptably high beam loss at injection and during the early part of the energy ramp. The modulated beam-beam effect due to different revolution frequencies of the two beams was the underlying cause 4 . The frequencies have been kept the same for both beams at injection and during the ramp to top energies for all the rest asymmetrical collision physics programs afterwards.

For proton collision with any fully stripped ions, it is challenging to match the frequencies of both beams. The DX magnets need to be moved to accommodate beam orbits, the ramp is specially designed to match injection frequencies [5]. For $\mathrm{p}-\mathrm{Au}(\mathrm{Al})$ run, $\mathrm{Au}(\mathrm{Al})$ beam is filled in the Yellow ring and ramped to cross transition and sitting at a porch near transition. At the same time mode switch back to proton. The proton beam was injected then to the Blue ring with the same revolution frequency. Both beams are accelerated together to the top energies with matched frequencies all along the ramp.

The increased complexity of the $\mathrm{p}-\mathrm{Au}(\mathrm{Al})$ operation motivated the revisit of accelerating beams with unequal frequencies. We did the experiments in both $\mathrm{p}-\mathrm{Au}$ and $\mathrm{p}-\mathrm{Al}$ run. The results of the study in $\mathrm{p}-\mathrm{Al}$ run will be presented here.

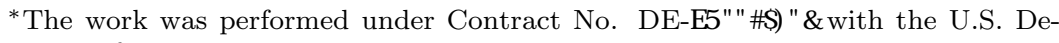
partment of Energy. 


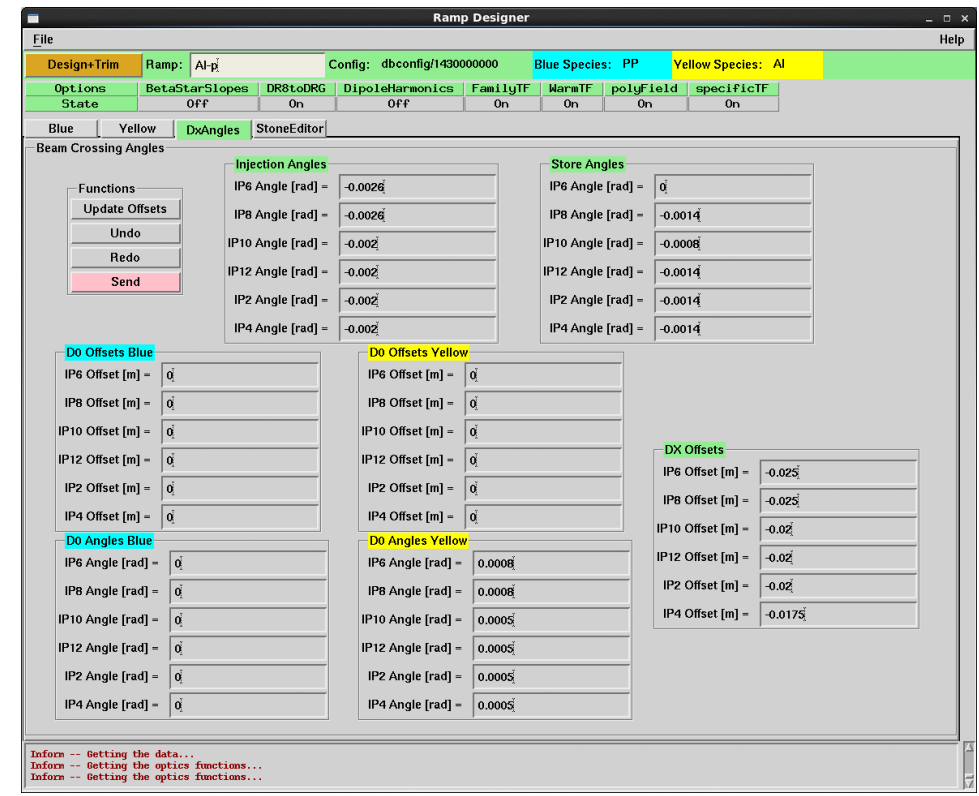

Figure 1: The crossing angles and D0 angles for the experimental ramp (Al-p). Beam crossing angles are defined separately for injection and store on the upper part. The D0 angles in Yellow are shown in the middle lower part. The DX offsets are the displacement of the DX magnets.

\section{Machine setup}

In order to avoid the cross talk between the common $9 \mathrm{MHz}$ cavity and the Yellow beam, the $28 \mathrm{MHz}$ cavity was employed for the Blue beam in the study. The frequency of the $9 \mathrm{MHz}$ cavity was kept $6 \mathrm{kHz}$ below the Yellow beam frequency. The revolution frequencies of the proton and the $\mathrm{Al}$ beam are respectively 78135.4155847 and $77950.4004115 \mathrm{~Hz}$.

The ramp design is based on the operation ramps for $\mathrm{p}-\mathrm{Al}$ run. As mentioned, the $\mathrm{Al}$ beam is injected in the Yellow ring first and accelerated alone to a intermediate energy. This part is the Alonly15-e0 ramp. The idea is to inject both beam at nominal energies at the same time and ramp the $\mathrm{Al}$ beam only to the intermediate energy. The ramp, Al-p, is a modified version of the Alonly15-e0 ramp.

It was necessary to employ some tricks to fit the beams with DX magnets moved. The DX magnets and other components were moved to accommodate the asymmetric beams with equal revolution frequencies and different beam rigidities (by factor of $\sim 2.5$ ). The beams in the study are at the nominal injection beam rigidities (79.4 $\mathrm{Tm}$ for proton, $81.1 \mathrm{Tm}$ for $\mathrm{Al}$ ). The crossing angles and D0 magnet angles were implemented to not only fit the beams in the available aperture, but also to keep the power supplies within the limits. 
The separation bumps were increased to reduce the beam-beam force for both beams. The size of the bumps in Blue ring are 8, -8, 10, 10, 10, $10 \mathrm{~mm}$ at IR6, 8, 10, 12, 2, and 4. The size of the bumps in Yellow ring are -7, 7, -9, -9, $-9,-9 \mathrm{~mm}$ at IR6, 8, 10, 12, 2, and 4 .

\section{Beam-beam for asymmetric beams}

In this section, the beam-beam force for asymmetric beams will be derived following the reference [6].

The charge distribution of the incoming beam (beam 2) is assumed Gaussian distribution. The transverse profile is round. The transverse charge distribution is given by:

$$
\rho(r)=\frac{N_{2} Q_{2}}{2 \pi \sigma^{2}} \exp \left(-\frac{r^{2}}{2 \sigma^{2}}\right)
$$

Applying Gauss's laws,

$$
2 \pi r E_{r}=\frac{1}{\epsilon_{0}} \int_{0}^{r} 2 \pi r^{\prime} \rho\left(r^{\prime}\right) d r^{\prime}
$$

one gets

$$
E_{r}=\frac{N_{2} Q_{2}}{2 \pi \epsilon_{0} r}\left(\exp \left(-\frac{r^{2}}{2 \sigma^{2}}\right)-1\right)
$$

Applying Ampere's laws,

$$
2 \pi r B_{\phi}=\mu_{0} \beta c \int_{0}^{r} 2 \pi r^{\prime} \rho\left(r^{\prime}\right) d r^{\prime}
$$

one gets

$$
B_{\phi}=\frac{\beta N_{2} Q_{2}}{2 \pi \epsilon_{0} r c}\left(\exp \left(-\frac{r^{2}}{2 \sigma^{2}}\right)-1\right)
$$

The beam-beam force experienced by particles in beam 1 is,

$$
\begin{gathered}
F=Q_{1}[E+v \times B] \\
=\frac{N_{2} Q_{1} Q_{2}}{2 \pi \epsilon_{0} r}\left(1+\beta^{2}\right)\left(\exp \left(-\frac{r^{2}}{2 \sigma^{2}}\right)-1\right)
\end{gathered}
$$

for $r<<\sigma$,

$$
F=\frac{N_{2} Q_{1} Q_{2} r}{4 \pi \epsilon_{0} \sigma^{2}}\left(1+\beta^{2}\right)
$$

for $r>>\sigma$,

$$
F=\frac{N_{2} Q_{1} Q_{2}}{2 \pi \epsilon_{0} r}\left(1+\beta^{2}\right)
$$

The beam-beam force due to the incoming bunch is the integration of the two dimensional force and the longitudinal density distribution of the incoming 
bunch. The beam-beam kick can be obtained from the integration of the beambeam force,

$$
\triangle r^{\prime}=\frac{N_{2} Q_{1} Q_{2}}{2 \pi \epsilon_{0} r m_{1} c^{2} \beta^{2} \gamma}\left(1+\beta^{2}\right)\left(1-\exp \left(-\frac{r^{2}}{2 \sigma^{2}}\right)\right)
$$

The inverse focal length of the beam-beam kick is $\delta=-\triangle r^{\prime} / r$, the incoherent beam-beam shift is,

$$
\begin{gathered}
\xi=\frac{\beta_{x} \delta}{4 \pi} \\
=-\frac{N_{2} Q_{1} Q_{2}\left(1+\beta^{2}\right) \beta_{x}}{(4 \pi)^{2} \epsilon_{0} m_{1} c^{2} \beta^{2} \gamma \sigma^{2}}
\end{gathered}
$$

Using $1 / \epsilon_{x}=\frac{\beta_{x}}{\beta \gamma \sigma^{2}}$, and $r_{0}=\frac{e^{2}}{4 \pi \epsilon m_{1} c^{2}}$,

$$
\epsilon=-\frac{N_{2} Q_{1} Q_{2} r_{0}}{4 \pi A_{1} \epsilon_{x}}
$$

In p-Au operation, $N_{p}=2.3 \times 10^{11}, N_{A u}=1.6 \times 10^{9}, \epsilon_{p}=2.5 \times 10^{-6}, \epsilon_{A u}=$ $3 \times 10^{-6}$. For $\mathrm{p}$-Au collision, the beam-beam parameter for proton beam is $5 \times 10^{-3} / \mathrm{IP}$, for $\mathrm{Au}$ beam is $4.4 \times 10^{-3} / \mathrm{IP}$.

In the beam study in p-Al, $N_{p}=1.8 \times 10^{11}, N_{A l}=1.2 \times 10^{10}$. The beambeam parameter for proton in head-on collision is $6.2 \times 10^{-3} / \mathrm{IP}$, for $\mathrm{Al}$ is $4.1 \times$ $10^{-3} / \mathrm{IP}$ assuming the same emittances as in p-Au.

The beta stars in both rings at injection are about $10 \mathrm{~m}$, and DX is 9.8 $\mathrm{m}$ away from the IPs. Therefore, the beta functions at DX magnet is roughly $20 \mathrm{~m}$. The RMS beam size of proton beam at injection is $\sigma_{p}=\sqrt{\left(\frac{\beta_{x} \epsilon}{\beta \gamma}\right)}=$ $\sqrt{\left(\frac{20 * 2.5}{25.4}\right)}=1.4 \mathrm{~mm}$. The RMS beam size of Aluminum beam at injection is $\sigma_{p}=\sqrt{\left(\frac{\beta_{x} \epsilon}{\beta \gamma}\right)}=\sqrt{\left(\frac{20 * 3}{12.6}\right)}=2.2 \mathrm{~mm}$.

The beam separation is roughly 10 times beam size, which drops the beambeam parameter by a factor of 0.04 [6]. Considering there are long range beam beam in all 6 IRs, the beam-beam tune shifts are $1.55 \times 10^{-3}$ for proton and $1.02 \times 10^{-3}$ for $\mathrm{Al}$ beam. A tune shift of that value is not large enough to cause large beam losses. However, a tune modulation depth at this level caused major beam losses in several experiments [7, 8, 9,

The difference in the revolution time of the two beams is $\triangle T=1 / f_{2}-1 / f_{1}=$ $3.0376^{-8}$. The time it takes for the same bunches to meet again next turn is $T 1+\triangle T / 2=T 2-\triangle T / 2$. The crossing point moves by $c \triangle T / 2$ during one turn 4. The distance from DX on one side to DX magnet on the other side of IP is $\triangle L=20 \mathrm{~m}$, which is the distance the two bunches can see one another. Therefore, two bunches will experience the beam-beam force from one another for $\triangle L /(c \triangle T / 2)=4$ turns. For the only bunch in one of the ring, the time it takes to slip into interaction with the next bunch in the other ring is $L / 120 /(c \triangle T / 2)=7$ turns. For the bunches in the full ring. the time it takes to come back into interaction with the only bunch in the other ring after a slippage of one sextant of the ring is $L / 6 /(c \triangle T / 2)=140$ turns. 


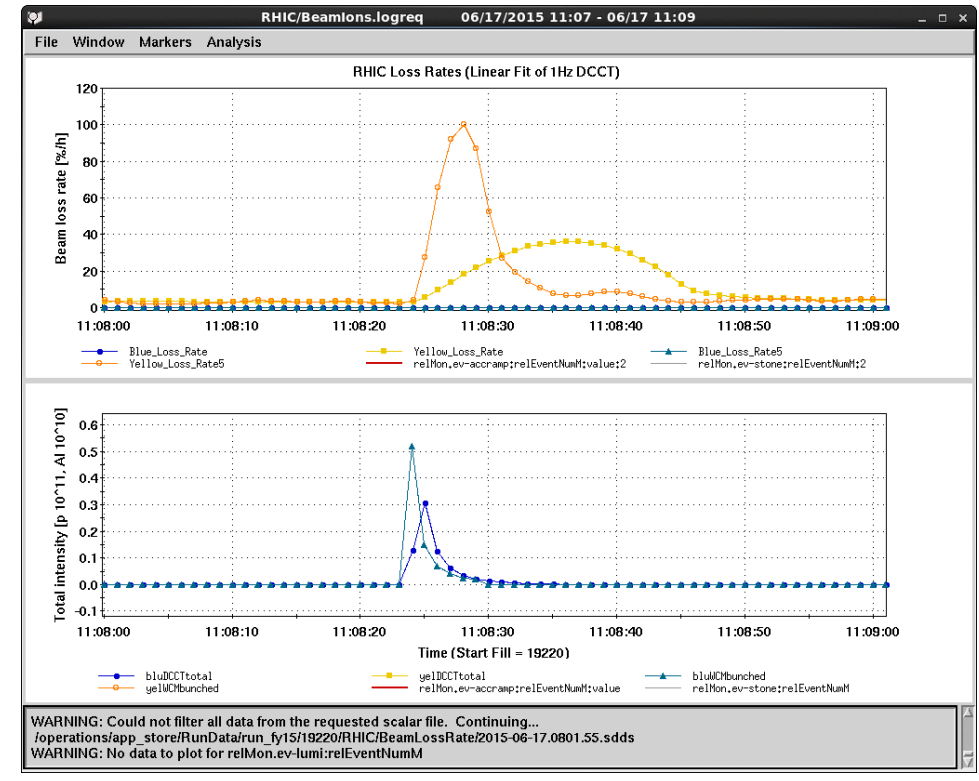

Figure 2: Yellow (Al) beam loss when inject one Blue (proton) bunch. The Yellow ring was filled with 111 bunches with $1.2 \times 10^{10} \mathrm{ppb}$.

\section{Observation during the study}

We were able to fill the Yellow ring with $\mathrm{Al}$ bunches first, and injected proton in the Blue ring. The one proton bunch suffered severe beam loss that the intensity drop right away (Fig. 2).

After the Blue was filled with proton bunches, we started to inject one $\mathrm{Al}$ bunch in the Yellow ring. The loss of the $\mathrm{Al}$ bunches intensity was less severe (Fig. 3).

Fig. 4 shows the beam loss pattern around the ring.

The beam loss and emittance blow-up was so severe that no further beambeam observation was possible.

\section{Acknowledgements}

The authors would like to thank A. Drees, C. Montag, M. Blaskwicz and W. Fischer for helpful discussions.

\section{References}

[1] T. Satogata, L. Ahrens, M. Bai, J. Beebe-Wang, M. Blaskiewicz, JM. Brennan, KA. Brown, D. Bruno, PP. Cameron, J. Cardona, et al. Commissioning 


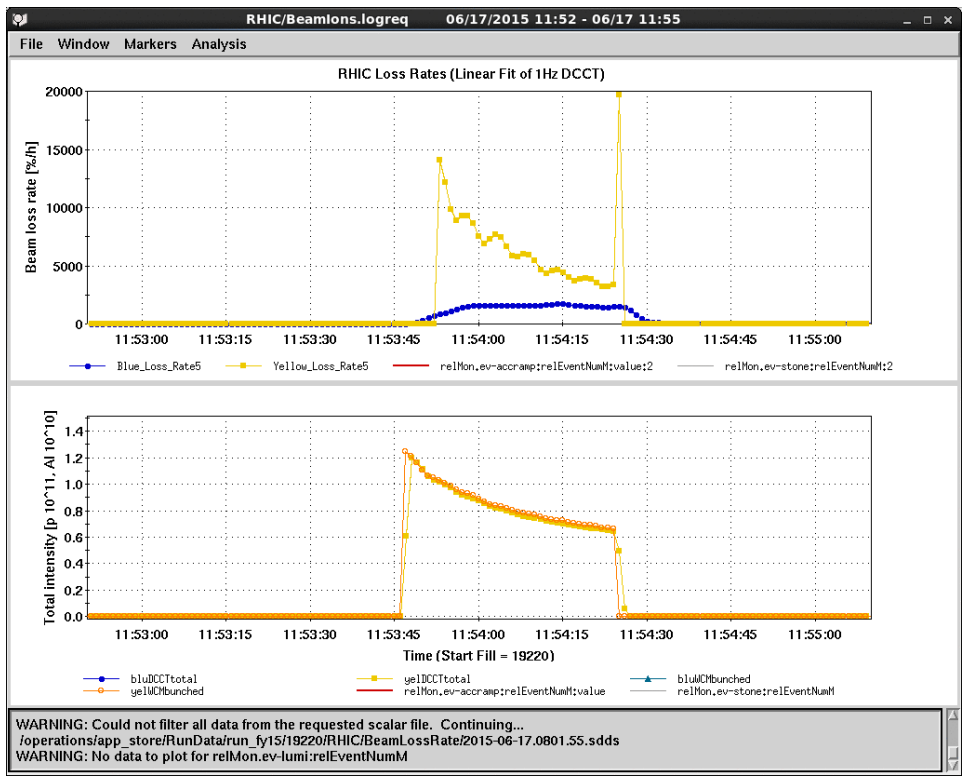

Figure 3: Blue (proton) beam loss when inject one Yellow (Al) bunch. The Blue ring was filled with 111 bunches with $1.8 \times 10^{11} \mathrm{ppb}$.

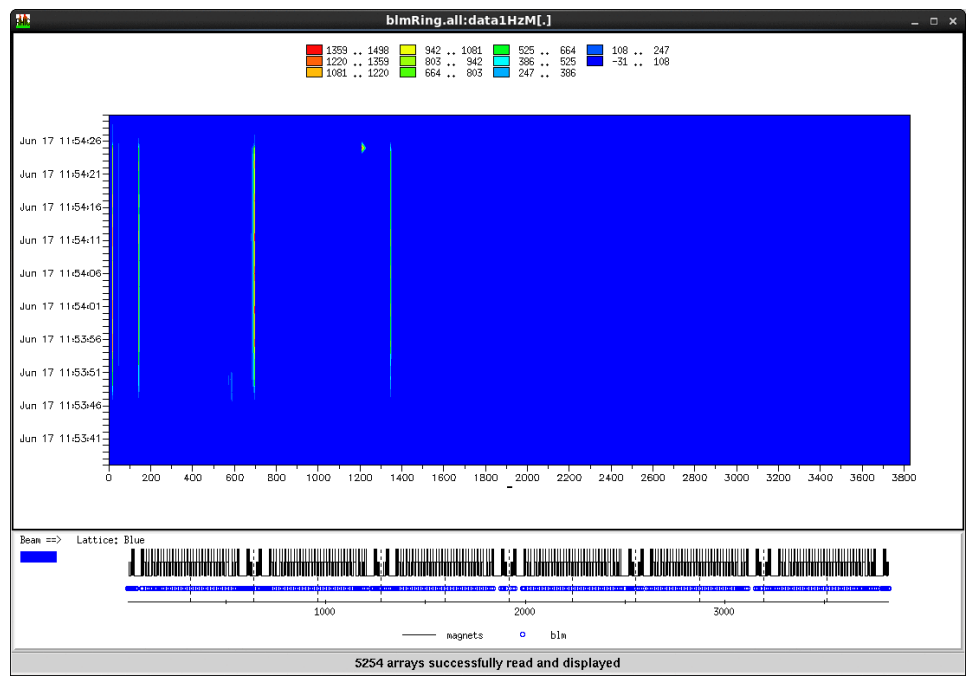

Figure 4: The beam loss pattern around RHIC. From left to right, the first line is beam loss at IR6, the second line is beam loss around b06-qd9, the third line is beam loss at the Yellow collimator, the fourth line is beam loss at the Blue dump. 
of RHIC deuteron-gold collisions. In Particle Accelerator Conference, 2003. PAC 2003. Proceedings of the, volume 3, pages 1706-1708. IEEE, 2003.

[2] W. Fischer. RHIC with asymmetric ion beams. Seminar at CERN, 2011.

[3] C. Liu. Challenging exotic operating modes in RHIC. Presentation at RHIC/AGS users' meeting, 2015.

[4] W. Fischer, P. Cameron, S. Peggs, and T. Satogata. Tune modulation from beam-beam interaction and unequal radio frequencies in RHIC. In AIP Conference Proceedings, pages 252-255, 2003.

[5] C. Liu. Preparations for p-Au run in 2015. Technical report, C-A/AP/532, 2014.

[6] E. Keil. Beam-beam dynamics. Technical report, CERN, 1994.

[7] W. Fischer, M. Giovannozzi, and F. Schmidt. Dynamic aperture experiment at a synchrotron. Physical Review E, 55(3):3507, 1997.

[8] T. Satogata, T. Chen, B. Cole, D. Finley, A. Gerasimov, G. Goderre, M. Harrison, R. Johnson, I. Kourbanis, C. Manz, et al. Driven response of a trapped particle beam. Physical review letters, 68(12):1838, 1992.

[9] O. Bruning and F. Willeke. PA 55, 237 (1996). 